\title{
Long-term quality of life profile in oncology: a comparison between cancer survivors and the general population
}

\author{
Maria Antonietta Annunziata ${ }^{1}$ Barbara Muzzatti $^{1}$ C Cristiana Flaiban $^{1}$. \\ Katiuscia Gipponi ${ }^{1}$ - Carlo Carnaghi ${ }^{2}$ - Paolo Tralongo ${ }^{3}$ - Michele Caruso ${ }^{4}$. \\ Raffaele Cavina $^{5} \cdot$ Umberto Tirelli $^{1}$
}

Received: 28 March 2017 / Accepted: 10 September 2017 / Published online: 16 September 2017

(C) Springer-Verlag GmbH Germany 2017

\begin{abstract}
Purpose Understanding the quality of life (QoL) of cancer survivors is relevant to both clinical practice and health care policy. The current study compared the QoL profile in this specific population with that of a normative sample for the general population, as well as with those of both healthy and oncological patients normative sub-samples. In addition, associations between the obtained QoL profile and the main socio-demographic and clinical characteristics of the sample were examined.

Methods Three hundred and ninety-two adult long-term cancer survivors (i.e., people $5+$ years from their cancer diagnosis who were free from it and its treatments) were enrolled during follow-up visits and compiled the Short Form 36 Health Survey.

Results In comparison with the normative data for the adult general population, the present sample showed lower scores in Physical functioning, Role-physical limitation, and Roleemotional limitations (all differences were both statistically and clinically significant); the difference in Vitality was only statistically significant. In all eight SF-36 scales, scores of the
\end{abstract}

Maria Antonietta Annunziata and Barbara Muzzatti contributed to the manuscript in equal part, thus they share first co-authorship.

Maria Antonietta Annunziata

annunziata@cro.it

1 Centro di Riferimento Oncologico, IRCCS Istituto Nazionale Tumori, Via F. Gallini, 2, 33081 Aviano, PN, Italy

2 Humanitas Mater Domini, Castellanza, Italy

3 Rete Assistenza Oncologica, Siracusa, Italy

4 Humanitas Centro Catanese di Oncologia, Catania, Italy

5 Humanitas Cancer Center, Rozzano, Italy present sample were clinically and statistically lower than those of the normative healthy subsample, whereas they were statistically and clinically higher than those of normative subsample which had experienced cancer, except for Rolephysical limitation. The QoL profile was associated with gender $(p=0.002)$, age $(p=0.001)$, education $(p<0.001)$, occupational status $(p<0.001)$, and the presence of other health issues $(p<0.001)$.

Conclusion These data support the utility of rehabilitative programs which integrate both healthcare and social interventions. In addition, they encourage the monitoring of the health status of this specific population, within a broad frame which simultaneously takes into consideration health and QoL.

Keywords Cancer survivorship · Health status · Neoplasm · Oncology $\cdot$ Quality of life $\cdot$ Well-being

\section{Introduction}

Cancer survivors are an increasing population which was only recently targeted by both clinicians and researchers, consequently, information on health and quality of life (QoL) of it is useful. In particular, more details on this topic are relevant to daily clinical practice, since they help to support individual patients, as well as health care policies, since they provide important information for the organization of services tailored on user needs.

In 2015, Annunziata et al. [1] assessed QoL (physical and mental functioning) of cancer survivors who have been free from the disease and its treatments for at least 5 years (i.e., long-term cancer survivors), demonstrating that in terms of QoL, long-term cancer survivors were different from both cancer patients and the general population. More specifically, they found that the average scores of physical and mental functioning were higher in their sample of long-term cancer survivors 
than those of cancer patients, but lower than those of the general population. Although these results sound interesting, further data are necessary to strengthen their clinical implications (i.e., to support the relevance and the rationality of clinical programs and services specifically dedicated to this population). In particular, since QoL is a multidimensional construct [2], using more comprehensive measures of QoL could be more informative and could help in better depicting both the health and functioning of this population. The literature provides evidence that being a cancer survivor very often means having to face late-presenting and/or long-term effects, both psychosocial and physical, that can impact different QoL domains years after treatment ends. Musculoskeletal problems, pain, sexual dysfunction, fatigue, anxiety, depressive symptoms, and cognitive limitations are some of the effects which can be found during treatment, and which can also affect long-term cancer survivorship [3-6]. More specific long-term effects such as relapses, iatrogenic tumors, fear of cancer recurrence, and limitations in restarting one's pre-illness functioning, are frequently denounced by survivors [3-5].

This study expanded knowledge on the QoL in long-term cancer survivors by means of a cross-sectional survey utilizing a multidimensional validated questionnaire (the SF-36) commonly used in different clinical settings as well as for general populations. In particular, it aims to compare QoL profile in this specific population with that of a normative sample for the general population, as well as with those of both healthy and oncological patients normative sub-samples. A further study's aim was to explore associations between the obtained QoL profile and the main socio-demographic and clinical characteristics of the sample.

\section{Methods}

\section{Participants}

The research involved long-term cancer survivors, i.e., people $5+$ years from their cancer diagnosis who were free from cancer and its treatments. Besides being a long-term cancer survivor, participants also had to meet the following inclusion criteria: age $\geq 18$ years, a good understanding of the Italian language, and provided informed consent. Prior mental disorders and physical or sensory disabilities that would affect the ability to fill out the forms were exclusion criteria for this study.

Less than $10 \%$ of approached eligible participants declined their participation.

\section{Materials and procedure}

All participants completed the Short Form 36 Health Survey Questionnaire (SF-36, [7]) and a form to collect sociodemographic and clinical data.
The SF-36 [7] is a self-report questionnaire which has been widely used with different populations (both healthy and clinical) for assessing QoL. It consists of 36 items providing eight different QoL indices: Physical functioning, Role-physical limitation, Bodily pain, General health, Vitality, Social functioning, Role-emotional limitations, and Mental health. Each index is ranged from 0 to 100 where higher scores correspond to better functioning in that domain. Reliability (internal consistency and temporal stability) of each SF-36 sub-scale was found to be adequate across different studies [7, 8]. Apolone et al. [8] provided both validation and norms (based on a sample representative of the Italian adult population) for the Italian version.

The socio-demographic and clinical data form allowed for the collection of information on gender, age, education, marital status, occupational status, cancer type, length of survivorship, and presence of other (than cancer) health problems.

Eligible participants were approached by a psychologist during follow-up visits. After explaining the purposes and methods of the research and answering all questions, signed informed consent was obtained. Participants received a survey booklet and a pre-paid envelope to return it. They were instructed to fill out materials at home and to return them by mail within 3 weeks. A reminder phone call was made 4 weeks after the mailing date to participants who had not returned the survey materials.

This study received the approval of the qualified Independent Ethics Committee.

\section{Statistical analysis}

Descriptive statistics for the eight sub-scales included in the SF-36 was calculated.

Three series of eight One-Sample $t$ test were performed to compare QoL scores with Italian norm mean values reported for the general population $(N=2031)$, healthy participants $(N=608)$, and cancer patients $(N=34)$ [8].

To test the association between QoL domains and the socio-demographic and clinical characteristics - gender, age ( $<50,50-59,60-69,70+$ years), education (compulsory vs. post-compulsory), work (gainfully employed or not), relationship status (partnered or not), cancer type (breast, colon-rectum, lymphoma, uro-genital, and other cancers), length of survivorship (5-9 vs. 10 + years), and other reported health issues (yes or. no) — a series of eight multivariate analysis of variance (MANOVA) was performed. When a factor was statistically significant (principal effect), ANOVAs (one for each QoL domain) were performed.

In all analyses, $p<0.05$ (two-tailed) was considered to be statistically significant. Bonferroni's correction was used, where appropriate, to avoid errors due to multiple comparisons. Regarding clinical and social significance, on a scale of 0-100, a difference of 5 units was considered significant [8]. 
All statistical analyses were carried out using SPSS software, Version 20 (SPSS Inc., Chicago, Ill.).

\section{Sample size}

The Italian SF-36 manual [8] establishes the sample necessary to individuate a five-point difference between the average of a group and reference norm which is between 104 (for Mental health scale) and 366 (for Role-physical limitation), considering alpha $=0.05$ and power $=80 \%$ and applying a two-tailed $t$ test.

\section{Results}

\section{Sample socio-demographic and clinical characteristics}

Out of the 408 eligible participants, 16 were dropped from the study since they provided an incomplete SF-36 questionnaire. Consequently, the final sample comprised 392 participants, $77.6 \%$ of whom were female. The median age was 60 years (range 28-88), and $47.2 \%$ of the sample had a post-compulsory education (i.e., had attended school for more than 8 years). The majority of participants in the sample had a non-employed status of retired, homemaker, or unemployed $(67.3 \%)$ and had a stable emotional relationship, noted as married or cohabiting $(64.4 \%)$. In terms of clinical data, the most common cancer diagnosis was breast cancer $(61.2 \%)$, followed by lymphoma (16.8\%), colorectal cancer $(7.1 \%)$, and genito-urinary cancer (5.4\%), whereas the remaining $9.4 \%$ of the sample was diagnosed with other cancer types. The median survivorship was 10 years (range 5-33), and $43.1 \%$ of the sample was reported as other (than cancer) health issues.

\section{Comparisons with normative data from Italian general population}

Table 1 shows average and standard deviations for the eight SF-36 subscales; it also shows the difference from the norm represented by adult Italian general population. As can be observed from the table with respect to the norm, the sample presented lower scores in Physical functioning, Role-physical limitation, Vitality, and Roleemotional limitations subscales. All differences were statistically and clinically significant apart from data on Vitality, which was statistically but not clinically significant. Differences from norm for Bodily pain, General health, Social functioning, and Mental health subscales were not significant.
Table 1 SF-36 scores: comparisons with normative data for Italian general population [8]

\begin{tabular}{lllcr}
\hline & M & SD & Mean difference & \multicolumn{1}{l}{$p$} \\
\hline PF & 78.19 & 23.84 & -6.27 & $<0.001$ \\
RP & 66.52 & 41.20 & -11.69 & $<0.001$ \\
BP & 70.52 & 26.85 & -3.15 & 0.021 \\
GH & 62.09 & 22.91 & -3.13 & 0.007 \\
VT & 58.47 & 19.11 & -3.42 & $<0.001$ \\
SF & 75.18 & 22.30 & -2.25 & 0.046 \\
RE & 69.00 & 38.97 & -7.16 & $<0.001$ \\
MH & 68.96 & 17.54 & 2.37 & 0.008 \\
\hline
\end{tabular}

$P F$ Physical functioning, $R P$ Role-physical limitation, $B P$ Bodily pain, $G H$ General health, $V T$ Vitality, $S F$ Social functioning, $R E$ Role-emotional limitations, $M H$ Mental health

\section{Comparisons with Italian normative data from both healthy and oncological populations}

Table 2 reports average and standard deviations for the eight SF-36 subscales; it also reports the average differences with respect to Italian norm both for the subsample which declared to be healthy and to the one with oncological experience.

For all eight SF-36 sub scales, the average differences between the present sample and healthy subsample were statistically and clinically significant, and reported a worse quality of life in the present sample with respect to reference data in all eight of the investigated domains.

Instead, in comparison with the oncological subsample normative data, the scores of the present samples were higher (both statistically and clinically significant) in all domains investigated except for Role-physical limitation.

\section{Association between QoL domains and socio-demographic and clinical characteristics}

A series of eight MANOVAs have been conducted to verify the association of each registered socio-demographic and clinical characteristic with the QoL domains together. In particular, gen$\operatorname{der}(p=0.002)$, age $(p=0.001)$, education $(p<0.001)$, occupational status $(p<0.001)$, and the presence of other health issues $(p<0.001)$ were found to be statistically associated, whereas relational status ( $p=0.190)$, cancer type $(p=0.244)$, and length of survivorship ( $p=0.879$ ) were not. Table 3 summarizes QoL scores according to the significantly associated factors. In particular, females reported lower Physical functioning $(p=0.001)$, higher Bodily pain $(p<0.001)$, lower Vitality $(p=0.001)$, and a worse Mental health $(p=0.002)$ than males (all these differences were also clinically significant). In both Physical functioning $(p<0.001)$ and Role-physical limitation $(p=0.003)$, there were statistically significant differences according to age groups: in fact in both domains, younger participants displayed higher 
Table 2 SF-36 scores (means and [standard deviations]): comparisons with normative data for both Italian healthy subsample and Italian cancer patients subsample [8]

\begin{tabular}{lrrrrrr}
\hline & & \multicolumn{2}{c}{ Italian healthy population } & \multicolumn{2}{c}{ Italian cancer patients } \\
& M & SD & Mean difference & $\boldsymbol{p}$ & Mean difference & $\boldsymbol{p}$ \\
PF & 78.19 & 23.84 & -19.11 & $<0.001$ & 17.59 & $<0.001$ \\
RP & 66.52 & 41.20 & -27.78 & $<0.001$ & 4.12 & 0.049 \\
BP & 70.52 & 26.85 & -18.68 & $<0.001$ & 13.52 & $<0.001$ \\
GH & 62.09 & 22.91 & -18.11 & $<0.001$ & 17.19 & $<0.001$ \\
VT & 58.47 & 19.11 & -13.73 & $<0.001$ & 10.17 & $<0.001$ \\
SF & 75.18 & 22.30 & -11.22 & $<0.001$ & 11.08 & $<0.001$ \\
RE & 69.00 & 38.97 & -19.00 & $<0.001$ & 10.40 & $<0.001$ \\
MH & 68.96 & 17.54 & -6.84 & $<0.001$ & 19.06 & $<0.001$ \\
\hline
\end{tabular}

$P F$ Physical functioning, $R P$ Role-physical limitation, $B P$ Bodily pain, $G H$ General health, $V T$ Vitality, $S F$ Social functioning, $R E$ Role-emotional limitations, $M H$ Mental health scores than the oldest sub-sample ( $p<0.001$; clinically significant differences). In comparison with their counterparts ( $p<0.001$; clinically significant differences), more educated participants reported higher Physical functioning, less Rolephysical limitation, less Bodily pain, and better General health. Employed participants reported a higher Physical functioning $(p<0.001)$, less Role-physical $(p<0.001)$ and Emotional-role $(p<0.001)$ limitations, less Bodily pain $(p<0.001)$, and a better General health $(p=0.002)$ than non-employed participants (all these differences were clinically significant). Finally, participants declaring no other health issues reported a higher Physical $(p<0.001)$ and Social $(p<0.001)$ functioning, less Role-physical $(p<0.001)$ and Role-emotional $(p=0.004)$ limitations, less Bodily pain $(p<0.001)$, a higher General health

Table 3 QoL profile (as displayed by SF-36) according to the associated socio-demographic/clinical characteristics (means and [standard deviations])

\begin{tabular}{|c|c|c|c|c|c|c|c|c|c|}
\hline & $\mathrm{N}$ & $\mathrm{PF}$ & $\mathrm{RP}$ & $\mathrm{BP}$ & GH & VT & $\mathrm{SF}$ & $\mathrm{RE}$ & $\mathrm{MH}$ \\
\hline \multicolumn{10}{|l|}{ Gender } \\
\hline Male & 88 & $85.68(19.45)$ & $76.42(36.62)$ & $81.25(24.67)$ & $67.76(22.04)$ & $64.60(18.53)$ & $80.03(20.58)$ & $76.02(36.15)$ & $74.14(14.43)$ \\
\hline Female & 304 & $76.02(24.58)$ & $63.65(42.05)$ & $67.41(26.70)$ & $60.45(22.93)$ & $56.70(18.94)$ & $73.77(22.61)$ & $66.96(39.57)$ & $67.46(18.09)$ \\
\hline$p$ & & 0.001 & 0.010 & 0.000 & 0.008 & 0.001 & 0.020 & 0.055 & 0.002 \\
\hline \multicolumn{10}{|l|}{ Age (years) } \\
\hline$<50$ & 83 & $86.08(20.26)$ & $80.12(34.43)$ & $75.76(26.83)$ & $66.22(20.52)$ & $59.16(19.94)$ & $73.36(24.32)$ & $75.35(34.65)$ & $66.51(17.40)$ \\
\hline $50-59$ & 111 & $79.73(22.44)$ & $67.57(39.54)$ & 70.95 (26.79) & $62.49(24.33)$ & $59.10(19.26)$ & $77.03(22.33)$ & $68.93(38.90)$ & $69.33(18.56)$ \\
\hline $60-69$ & 114 & $77.72(22.16)$ & $63.38(42.36)$ & $67.11(26.78)$ & $62.44(21.42)$ & $59.32(18.37)$ & $76.75(20.11)$ & $69.46(38.86)$ & $71.12(15.69)$ \\
\hline $70+$ & 81 & 69.57 (28.09) & $57.10(44.59)$ & $70.67(26.27)$ & $57.37(24.47)$ & $56.42(19.22)$ & $73.23(22.89)$ & $63.27(42.72)$ & $68.15(18.76)$ \\
\hline$p$ & & 0.000 & 0.003 & 0.170 & 0.101 & 0.716 & 0.480 & 0.266 & 0.313 \\
\hline \multicolumn{10}{|l|}{ Education (years) } \\
\hline$<9$ & 205 & $73.15(24.82)$ & $56.95(42.84)$ & $65.15(27.11)$ & $58.18(23.49)$ & $56.62(19.59)$ & $74.17(21.24)$ & $65.06(39.56)$ & $69.54(17.81)$ \\
\hline $9+$ & 183 & 83.66 (21.64) & $76.91(36.73)$ & $76.14(25.52)$ & $66.32(21.61)$ & $60.33(18.41)$ & $76.11(23.64)$ & $73.10(38.08)$ & $68.09(17.33)$ \\
\hline$p$ & & 0.000 & 0.000 & 0.000 & 0.000 & 0.056 & 0.393 & 0.043 & 0.417 \\
\hline \multicolumn{10}{|l|}{ Occupational status } \\
\hline Employed & 124 & $85.93(19.70)$ & $85.08(29.72)$ & $76.94(24.79)$ & $66.96(22.69)$ & $61.53(18.99)$ & $78.58(23.08)$ & $81.60(31.43)$ & $70.23(16.86)$ \\
\hline Non-employed & 255 & $73.98(24.87)$ & $56.76(43.20)$ & $66.57(27.31)$ & $59.20(22.57)$ & $56.87(18.78)$ & $73.45(21.61)$ & $61.69(41.00)$ & $68.25(18.16)$ \\
\hline$p$ & & 0.000 & 0.000 & 0.000 & 0.002 & 0.024 & 0.035 & 0.000 & 0.310 \\
\hline \multicolumn{10}{|c|}{ Presence of other health issues } \\
\hline Yes & 169 & $71.15(26.02)$ & $55.77(43.39)$ & $60.91(26.92)$ & $54.00(23.92)$ & $52.41(19.21)$ & $68.63(26.00)$ & $62.57(40.51)$ & $66.46(18.73)$ \\
\hline No & 223 & $83.52(20.56)$ & $74.66(37.56)$ & $77.80(24.46)$ & $68.22(20.10)$ & $63.07(17.74)$ & $80.14(17.53)$ & $73.87(37.11)$ & $70.85(16.37)$ \\
\hline$p$ & & 0.000 & 0.000 & 0.000 & 0.000 & 0.000 & 0.000 & 0.004 & 0.014 \\
\hline
\end{tabular}

$P F$ Physical functioning, $R P$ Role-physical limitation, $B P$ Bodily pain, $G H$ General health, $V T$ Vitality, $S F$ Social functioning, $R E$ Role-emotional limitations, $M H$ Mental health 
$(p<0.001)$, and more Vitality $(p<0.001)$ than their counterparts (all these differences were clinically significant).

\section{Discussion}

With respect to the general adult population, this sample consists of 392 Italian adults who have obtained a diagnosis of malignancy for at least 5 years and who are free of disease and treatments, showed: worse Physical functioning; greater role limitations, much due to physical and emotional problems; and less Vitality (although in this case, the statistical significance was only and non-clinical). In other words, with a relevance that is both clinical and social [8], the participants in this study reported more limitations in the exercise of physical activity because of their health (Physical functioning), greater difficulties (i.e., limitations, reduction of dedicated time, and/or difficulties in the implementation) with the work and/or other daily activities due to both physical health (Role-physical limitation), and emotional problems (Role-emotional limitation). However, people in this sample did not diverge from the general population in terms of intensity of physical pain and its interference in daily life (Bodily pain); current health, resistance to disease, and vision of health (General health); effects of both the physical health because of emotional problems on social activities (Social functioning); and Mental health. In France, Klein et al. [9] compared the quality of life measured by the SF36, the long-term cancer survivors (their sample was, however, made up only of women with a history of breast cancer) and controls (stratified for age and place of residence, randomly selected from electoral rolls), with poorer scores seen in the long-term cancer survivors in all eight domains, except for Mental health. Furthermore, as in the present study, the domains for which the mean differences were clinically relevant were Physical functioning, Role-physical limitation, and Role-emotional limitation. Worse SF-36 scores except Bodily pain in a sample of UK cancer survivors (heterogeneous for cancer type and survivorship length) than in controls from the general population (matched by gender and age) were also found by Santin et al. [10]. Conversely, Helgeson and Tomich [11] compared the SF-36 scores of 5 years diseasefree breast cancer survivors with those of age- and neighborhood-matched controls and obtained statistically significant differences only in Physical functioning (lower in survivors than controls) (see also [12]).

In addition to the comparison with the general population, in the present study, comparison with standard data for healthy adults and comparison with standard data for cancer patients were carried out. Comparing with the norm on the subsample is declared healthy adult (i.e., without self-reported medical conditions) [8], the present sample showed clinically and socially worse scores in all eight domains investigated. At the same time, in comparison with the normative sub-sample, made up of people who have or have had cancer or a malignant tumor, the present sample showed better scores in all of the investigated domains, except for Role-physical limitation (not moving away, so from oncology normative group for problems with the work and/or other daily activities due to physical health). These data can be combined with those of Heins et al. [13], who compared the QoL of a sample of cancer survivor adults for at least 2 years after diagnosis with that of a sample of chronic patients (for example suffering from diabetes mellitus, asthma/COPD, ischemic heart disease, arthritis): in the latter study, the average QOL of cancer survivors was better than that of patients with chronic diseases, even after controlling for gender, age, and time since diagnosis in all SF36 domains, with the exception of Mental health. Taken together, the data of the current study deepen and specify the information described above for a sample of Italian long-term cancer survivors [1]. In addition, they expand the results of these cross-sectional studies (e.g., [9-12, 14]) that show how the quality of life of the survivor with more years of diagnosis tends to approach that of the controls to a greater extent than is the case for survivor in a smaller number of years from diagnosis.

Finally, this study provides some useful information about the role of some socio-demographic and clinical moderators of the eight domains of QOL considered. In line with that which has already been described in the literature $[1,15,16]$, there are gender differences. In the present study, women showed worse Physical functioning, more Bodily pain, Vitality, and worse Mental health. The older subsample, compared to younger participants, displayed a worse Physical functioning and more Role-physical limitations (see also [1, 17, 18]); both findings can be interpreted in the light of the role played by the primary aging process $[19,20]$. Participants with better education and still working participants have both showed better Physical functioning, less Role limitations (due to physical or emotional problems), less Bodily pain, and better General health with respect to their counterparts. These data are not surprising; they expand and complement what was found in previous studies [1, 15, 21, 22]. For example, in a study involving 24,810 US adults with self-reported physician diagnosis of cancer and 382,837 US adults with no cancer history, Clarke et al. [22] showed how the non-working active cancer subsample, compared to those who claimed not to be, more often reported good or excellent health (although in a smaller percentage than the controls without any experience of cancer). Moreover, the percentage of functional limitations reported among not employed survivors was almost twice that of employed survivors, and more than twice that of not employed persons without a cancer history. Finally, the same study confirmed there was a positive association between education and health status. However, it should be mentioned as other studies (for example [18]) showed that education was not associated with QoL. Finally, as already described elsewhere $[1,12,15,17,18,23,24]$, those who reported having 
other health problems showed a worse quality of life in all domains considered (except in Mental health, in the present study).

The main limitation of the current study consists of the absence of data on specific anti-cancer treatments for each participant, including type and duration; consequently, different potential effects of them on QoL could not be accounted for. In addition, in interpreting these data, we should be aware that female participants were more numerous than males, and that breast cancer was the predominant diagnosis in the enrolled sample; these facts may have influenced the patterns observed. However, the large prevalence of participants with breast cancer, together with the long time from anticancer treatment might tend to minimize the long-term effects of treatment. Another major limitation was the absence of information about rehabilitative procedures (physical or psychological) that could have influenced the results. Finally, we must keep in mind that the Italian norms for the SF-36 dated back to 1997, and that the reference sample of Italian oncological patients is quite small. Conversely, the main study strength was represented by its power and by the good psychometric properties of the QoL measure used.

In summary, data provided by the current study show how the quality of life of long-term survivors is probably affected by cancer and its treatment. Further studies should assess the quality of life of these users monitoring and of well designed interventions.

Acknowledgements The authors wish to thank Ms. Anna Vallerugo, MA, for her editorial assistance, and Dr. Sara Busato and Dr. Marika Piccinin for their help in data entry.

Funding information This work was supported by Italian Ministry of Health: CCM "Valutazione della Qualità di Vita e degli aspetti psicosociali della Cancer Survivorship e ottimizzazione dei programmi di sorveglianza" (2012-2014).

\section{Compliance with ethical standards}

Conflict of interest The authors declare that they have no conflicts of interest.

\section{References}

1. Annunziata MA, Muzzatti B, Giovannini L, Romito F, Cormio C et al (2015) Is long-term cancer survivors' quality of life comparable to that of the general population? An Italian study. Support Care Cancer 23:2663-2668

2. World Health Organization, QOL GROUP (1993) Study protocol for the World Health Organization project to develop a quality of life assessment instrument (WHOQOL). Qual Life Res 2:153-159

3. Stein KD, Syrjala KL, Andrykowski MA (2008) Physical and psychological long-term and late effects of cancer. Cancer 112(11 suppl):2577-2592

4. Foster C, Wright D, Hill H, Hopkinson J, Roffe L (2009) Psychosocial implications of living 5 years or more following a cancer diagnosis: a systematic review of the research evidence. Eur J Cancer Care (Engl) 18:223-247

5. Harrington CB, Hansen JA, Moskowitz M, Todd BL, Feuerstein M (2010) It's not over when it's over: long-term symptoms in cancer survivors - a systematic review. Int J Psychiatry Med 40:163-181

6. Wu HS, Harden JK (2015) Symptom burden and quality of life in survivorship: a review of the literature. Cancer Nurs 38:E29-E54

7. Ware JE, Snow KK, Kosinski M, Gandek B (1993) SF-36 health survey-manual and interpretation guide. The Health Institute Boston, MA

8. Apolone G, Mosconi P, Ware JE (1997) Questionario sullo stato di salute SF-36, manuale d'uso e guida all'interpretazione dei risultati. Guerini e Associati, Milano

9. Klein D, Mercier M, Abeilard E, Puyraveau M, Danzon A, Dalstein Vet al (2011) Long-term quality of life after breast cancer: a French registry-based controlled study. Breast Cancer Res Treat 129:125134

10. Santin O, Mills M, Treanor C, Donnelly M (2012) A comparative analysis of the health and well-being of cancer survivors to the general population. Support Care Cancer 20:2545-2552

11. Helgeson VS, Tomich PL (2005) Surviving cancer: a comparison of 5-year disease-free BCS with healthy women. Psychooncology 14: 307-317

12. Schoormans D, Czene K, Hall P, Brandberg Y (2015) The impact of comorbidity on health-related quality of life in breast cancer survivors and controls. Acta Oncol 54:727-734

13. Heins MJ, Korevaar JC, Hopman PE, Donker GA, Schellevis FG, Rijken MP (2016) Health-related quality of life and health care use in cancer survivors compared with patients with chronic diseases. Cancer 122:962-970

14. Wikman A, Djärv T, Johar A, Lagergren P (2013) Health-related quality of life does not differ between short-term, long-term and very long-term cancer survivors in the Swedish general population. Psychooncology 22:1369-1374

15. Muzzatti B, Flaiban C, Surbone A, Annunziata MA (2015) Quality of life profile in Italian long-term cancer survivors. Qual Life Res 24:959-967

16. ESEMeD/MHEDEA 2000 Investigators (2004) Disability and quality of life impact of mental disorders in Europe: results from the European Study of the Epidemiology of Mental Disorders (ESEMeD) project. Acta Psychiatr Scan 109(Suppl. 420):21-27

17. Rodriguez JL, Hawkins NA, Berkowitz Z, Li C (2015) Factors associated with health-related quality of life among colorectal cancer survivors. Am J Prev Med 49(Suppl 5):S518-S527

18. Chu WO, Dialla PO, Roignot P, Poillot ML, Coutant C, Arveux P (2016) Determinants of quality of life among long-term breast cancer survivors. Qual Life Res 25:1981-1990

19. Laicardi C, Pezzuti L (2000) Psicologia dell'invecchiamento e della longevità [Psychology of ageing and longevity]. Il Mulino Bologna

20. De Beni R, Borella E (2016) Psicologia dell'invecchiamento e della longevità [Psychology of ageing and longevity]. Il Mulino Bologna

21. Lee SY, Kim SJ, Shin J, Han KT, Park EC (2015) The impact of job status on quality of life: general population versus long-term cancer survivors. Psychooncology 24:1552-1559

22. Clarke TC, Christ SL, Lee DJ, Arheart KL, Prado G, Martinez AC et al (2015) Working with cancer: health and employment among cancer survivors. Ann Epidemiol 25:832-838

23. Smith SK, Crespi CM, Petersen L, Zimmerman S, Ganz PA (2010) The impact of cancer and quality of life for posttreatment nonHodgkin lymphoma survivors. Psychooncology 19:1259-1267

24. Le Borgne G, Mercier M, Woronoff AS, Guizard AV, Abeilard E, Klein D et al (2013) Quality of life in long-term cervical cancer survivors: a population-based study. Gyn Oncol 129:222-228 